\title{
The Uncertain Energy of an Excited State
}

\author{
Richard A. Hutchin \\ Optical Physics Company, Calabasas, CA, USA \\ Email: rahutchin@opci.com
}

Received 18 March 2015; accepted 27 June 2015; published 30 June 2015

Copyright (C) 2015 by author and Scientific Research Publishing Inc.

This work is licensed under the Creative Commons Attribution International License (CC BY). http://creativecommons.org/licenses/by/4.0/

(c) †) Open Access

\begin{abstract}
This paper asks an experimental question about photon absorption and emission in a two-state electronic system where the incident frequency of exciting light is below the mean frequency of the emission line but still within the line width. Since it is well known that the absorption spectrum and the emission spectrum are identical [1]-[3], then quantum mechanics as well as astronomical observations say that excitations will still occur. Once a quantum state excites, then it is expected to decay back with a typical time constant and the usual emission spectrum. The problem is that the mean frequency of that emission spectrum will then be higher than the frequency of the exciting photon, suggesting that more energy is emitted than being received on average. A search of quantum mechanical references finds no discussion of this issue, so this paper considers the options available to resolve this inconsistency, including an experiment.
\end{abstract}

\section{Keywords}

Photonic Emission, Absorption, Lorentz Line Shape, Conservation of Energy

\section{Introduction}

It is well known that an excited electronic state emits a spectrum of light with bandwidth proportional to $1 / \tau$, where $\tau=$ the decay time constant of the excited state [3]. It is also well known that the curve of excitation crosssection versus wavelength has the same shape as the emission spectrum [1]-[3]. Conservation of energy in this simple scenario leads to a perplexing question, whose answer will impact our physical models of light-matter interaction. In our search, we have found no prior discussion of this potential energy imbalance, likely because it is small and relatively unimportant to most experiments. However, any true energy imbalance would be profound to physics.

\section{Discussion}

A heavy two state electronic system (such as used in Einstein’s 1917 paper on stimulated emission) [3] has a

How to cite this paper: Hutchin, R.A. (2015) The Uncertain Energy of an Excited State. Optics and Photonics Journal, 5, 201-204. http://dx.doi.org/10.4236/opj.2015.56019 
Lorentz emission spectrum [1] [2] [4] as shown in Figure 1. It also has exactly the same absorption spectrum, since emission and absorption spectra are identical [1]-[3]. The mean frequency $v_{0}$ of the Lorentz spectrum corresponds to emitting a photon whose energy exactly equals the energy change in the quantum electronic transition. Incident photons with frequencies below that peak have less energy than the electronic state and those above that frequency have more since the energy of a photon equals $h v$.

To explain the range of frequencies of light emitted from a fixed state transition, quantum theory tells us that the finite decay time of the excited state creates an uncertainty in the energy of the state, resulting in a spread of energies in the emitted and absorbed photons. Mathematically, the Fourier transform squared of the exponential decay amplitude produces the Lorentz line shape-commonly observed. [2] [4] Planck's constant h times the mean frequency is the energy difference between the two quantum levels.

This paper proposes an experiment shown in Figure 2 to explore more precisely the question of energy balance between the energy of the initial excitation and the energy of the photonic emission in the resulting electronic decay. Are they required to be equal or not? We see two possible answers:

$$
\mathrm{L}(v)=\frac{\left(\frac{\Delta v}{2}\right)^{2}}{\left(v-v_{0}\right)^{2}+\left(\frac{\Delta v}{2}\right)^{2}}
$$

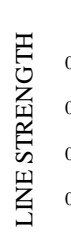
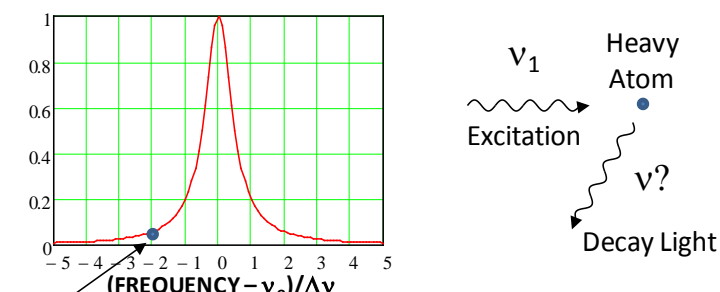

$\left(\right.$ FREQUENCY $\left.-v_{0}\right) / \Delta v$

Excitation

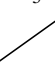

Frequency $v_{1}$

Figure 1. A heavy atom in an undisturbed environment decays with a lifetime $\tau$ that creates a Lorentz line shape $\mathrm{L}(v)$ - well known to physics. The mean wavelength $v_{0}$ of the Lorentz line is the expected transition frequency between the upper and lower quantum states. We ask what happens when the atom is illuminated by a monochromatic frequency below its mean frequency?

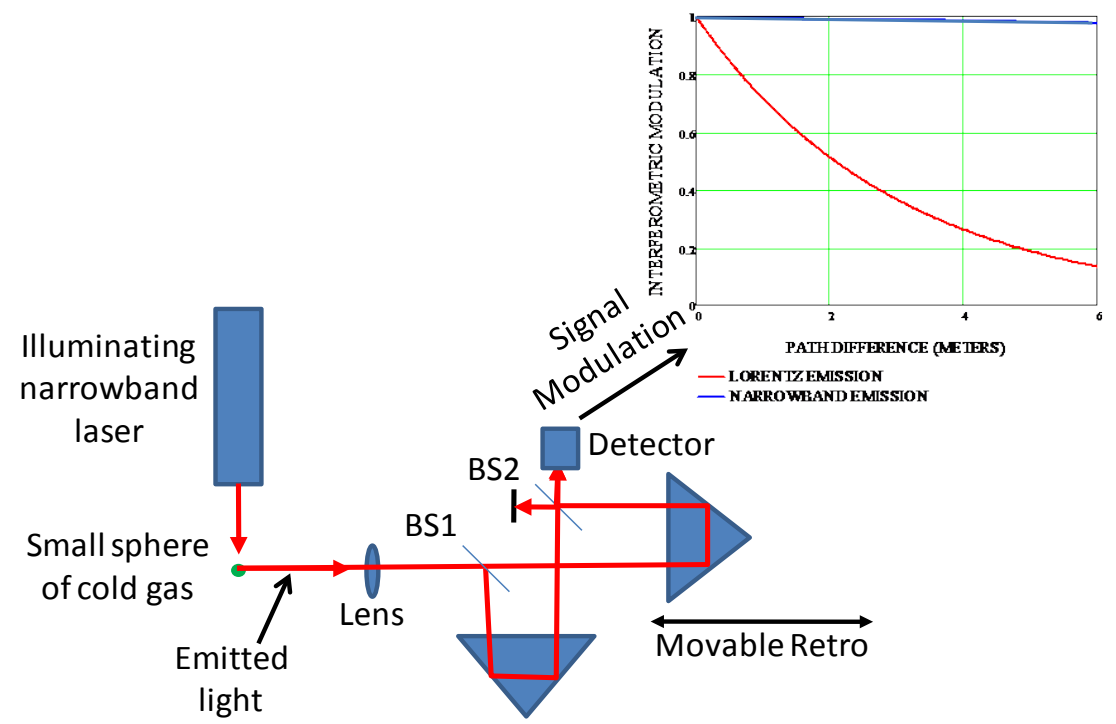

Figure 2. In the suggested experiment, a narrowband laser with wavelength below the mean Lorentz frequency illuminates a small volume of cold gas, which excites and then decays. The decay light is collimated by a lens and then passed to a Michelson type interferometer with a variable path length between the two paths. The modulation observed will either match the standard Lorentz emission spectrum (red curve) or the narrowband spectrum (blue curve). 
1) If they must be equal, then a precisely determined excitation energy should imply a precisely determined emission energy from the decaying state. This will change the resulting line shape to be much narrower than usual, resulting in much longer coherence length for the emitted light. This can easily be measured in a scanning interferometer as shown in Figure 2.

2) The other option is that the resulting emission after monochromatic excitation is unchanged compared to any other emission. This would mean that any excitation, no matter what frequency input, results in the same type of excited state-much simpler from that point of view. In this case the line shape of the emitted light will be the usual one determined by its standard decay time. However, if we consistently excite with a frequency $v_{\mathrm{ex}}$ below the mean Lorentz frequency $v_{0}$ while getting a mean frequency of $v_{0}$ re-emitted, then we must ask about energy conservation. How can we, on average, get $h\left(v_{0}-v_{\mathrm{ex}}\right)$ more energy emitted out than we put in?

\section{The Experiment}

In this experiment we irradiate a cold gas with a wavelength tuned to a narrow line of frequency $v_{1}$, which is below the mean frequency $v_{0}$ of the Lorentz line but within the width of the Lorentz line as shown in Figure 1. Then we look at the frequency distribution of the emitted light from the excited atom or molecule as shown in Figure 2. Typically this frequency is best measured using a scanning interferometer that determines the signal modulation of the emission as a function of the path difference as shown in Figure 2. The Fourier transform of this coherence function is the line shape. A $100 \mathrm{KHz}$ laser, will be coherent over $3 \mathrm{~km}$, while a typical atomic decay of 10 nanosec mean lifetime will have a 1/e coherence length of about 3 meters - 1000X shorter. Thus a coherence length measurement will easily tell the difference between a normal decay and a narrow band decay.

We note that this experimental configuration is very similar to Doppler cooling of atoms [5] [6] close to absolute zero, where a laser is tuned below the Lorentz line width and then progressively closer to the line center. The atoms absorb the light preferentially when they are moving toward the light source due to the Doppler shift, which slows them down. A lab setup like that could be modified to do this experiment by adding a scanning interferometer.

\section{Summary}

A simple question has been asked about the interaction between how an electron is excited and its resulting decay. Quantum mechanics does not contain such an interaction at present, but the absence of such an interaction leads to non-conservation of energy or an unknown process to balance the input and output energy. Since experiments always have the final word, it would be useful to know which path to follow.

\section{Conclusion}

This discussion was intended to motivate an experimental investigation of the excitation and emission of excited states. Does the emission depend at all on the excitation process? If not, how can we maintain conservation of energy? We could just as easily have focused on excitation energies larger than the mean value of the Lorentz spectrum and asked where the extra energy went. Answers of uncertainty will not suffice here, because the energy of an exciting photon of frequency $v_{\mathrm{ex}}$ is known to be exactly $h v_{\mathrm{ex}}$, and the mean energy of the emission back to the ground state is also accurately measured by the interferometer. By irradiating the atom with that known energy and measuring the frequency distribution of the emitted light, we can precisely determine energy balance or its lack. Either outcome will provide new insight into electronic transitions. Our hope is that an experimentalist with appropriate skills and equipment will provide the answer.

\section{References}

[1] Sharma, A. and Schulman, S.G. (1999) Introduction to Fluorescence Spectroscopy. John Wiley \& Sons, Chichester.

[2] Emission and Absorption Spectra. Department of Physics, University of Illinois. https://van.physics.illinois.edu/qa/listing.php?id=1910

[3] Line Width and Line Shape. www.pci.tu-bs.de/aggericke/PC4e/Kap_III/Linienbreite.htm

[4] Einstein, A. (1917) Zur Quantentheorie der Strahlung (On the Quantum Theory of Radiation). Physika Zeitschrift, 18, 121-128. 
[5] Wineland, D.J. and Dehmelt, H. (1975) Proposed 1014 $\Delta v<v$ Laser Fluorescence Spectroscopy on Tl + Mono-Ion Oscillator. Bulletin of the American Physical Society, 20, 637.

[6] Wineland, D.J., Drullinger, R.E. and Walls, F.L. (1978) Radiation-Pressure Cooling of Bound Resonant Absorbers. Physical Review Letters, 40, 1639. http://dx.doi.org/10.1103/PhysRevLett.40.1639 